research article

\title{
Budd-Chiari syndrome associated with liver hydatid disease: retrospective evaluation of color Doppler US findings with emphasis on intrahepatic venous collateralization
}

\author{
Cengiz Yilmaz ${ }^{1}$, Nazif Erkan², Muhammet Arslan', \\ Yusuf Yildirim ${ }^{2}$, Sena Kalaycioglu ${ }^{3}$ \\ ${ }^{1}$ Department of Radiology, \\ ${ }^{2}$ Department of General Surgery, Izmir Bozyaka Training and Research Hospital, Bozyaka, \\ ${ }^{3}$ Department of Radiology, Sifa Hospital, Basmane, Izmir, Turkey
}

\begin{abstract}
Background. The purpose of this retrospective study is to evaluate the color Doppler US (CDUS) findings of Budd-Chiari Syndrome (BCS) associated with hydatid disease of the liver (HDL) with a special emphasis on the intrahepatic venous collateralisation.

Methods. Digitally stored CDUS videoclips or videotape records of the liver of 13 patients with HDL and BCS were retrospectively reviewed. A special emphasis was placed on intrahepatic venous collaterals compatible with BCS.

Results. During the retrospective analysis of the sonographic data, at least one type of intrahepatic venous collateral typical for BCS was detected in all patients. CDUS revealed 5 different types of intrahepatic venous collaterals including subcapsular veins, comma-shaped veins which sometimes resemble hockey-stick, spider-web collaterals, fragmented veins and linear veins distributed in a non-anatomical fashion. The most frequently encountered collaterals at CDUS were comma-shaped veins, seen in 12 of 13 (92.3\%) patients. In addition, at least 2 different types of intrahepatic collaterals were found in 8 of 13 (61.5\%) patients. In two patients, venous collaterals were not seen on gray-scale sonography, but they were apparent at CDUS. Conclusions. BCS secondary to HDL may come as an unexpected finding during sonography. Familiarity with the typical sonographic appearances of the intrahepatic venous collaterals associated with BCS may enable the correct diagnosis and the prompt further treatment. We recommend CDUS to be performed in every patient with HDL regardless of the clinical presentation.
\end{abstract}

Key words: Budd-Chiari syndrome; liver hydatid disease; color Doppler sonography

Received 6 May 2009

Accepted 15 July 2009

Correspondence to: Dr. Cengiz Yilmaz, Izmir Bozyaka Training and Research Hospital, Department of Radiology, Saim Cikrikci Street, 35360, Bozyaka, İzmir, Turkey. Phone: +90 53247580 94; Fax: +90 232 26144 44; E-mail: cengizyilmaz66@yahoo.com 


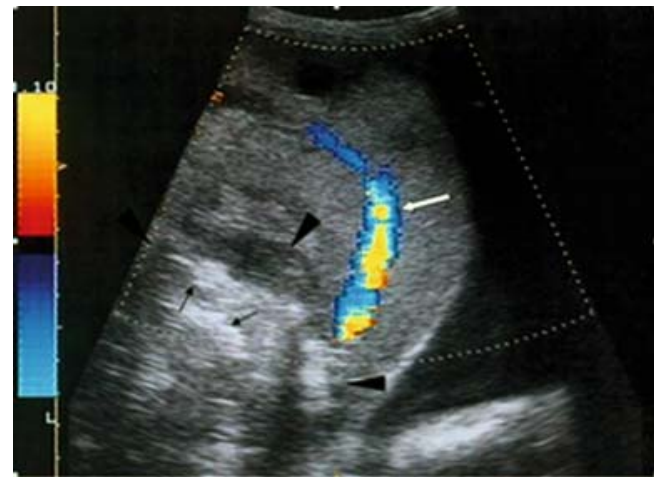

Figure 1A. A 37-year-old woman with HDL and BCS presented with right upper abdominal pain and fever. Sagittal color Doppler sonogram reveals a heterogeneous mass (arrowheads) including hyperechoic linear, serpentine structures (black arrows) representing degenerated and collapsed membranes. Also noted is a large subcapsular collateral vein (white arrow) which was shown to drain into the suprahepatic inferior vena cava at real time US.

\section{Introduction}

Budd-Chiari Syndrome (BCS) develops secondary to hepatic venous obstruction or interruption of the suprahepatic vena cava inferior (IVC). ${ }^{1,2}$ In the western world, the most common causes are prothombotic disorders like polycythaemia vera, whereas membranous obstruction is the leading cause in Asia. ${ }^{3,4}$ Hydatid disease of the liver (HDL) is a rare cause of BCS and is primarily encountered where the disease is endemic. ${ }^{5,6}$ The clinical presentation depends on the extent of the obstructive process, how quickly it develops and the degree of the intrahepatic collateralisation. ${ }^{7}$ The diagnosis of BCS is often missed unless its possibility has been kept in mind. ${ }^{8}$

Altough the association of BCS and HDL has been previously reported, most of these studies are either case reports or clinical studies without any significant imaging evidence. ${ }^{9-13}$ To the best of our knowledge, our study is the first in which this association is demonstrated at US in a relatively high number of patients. The aim of this retro-

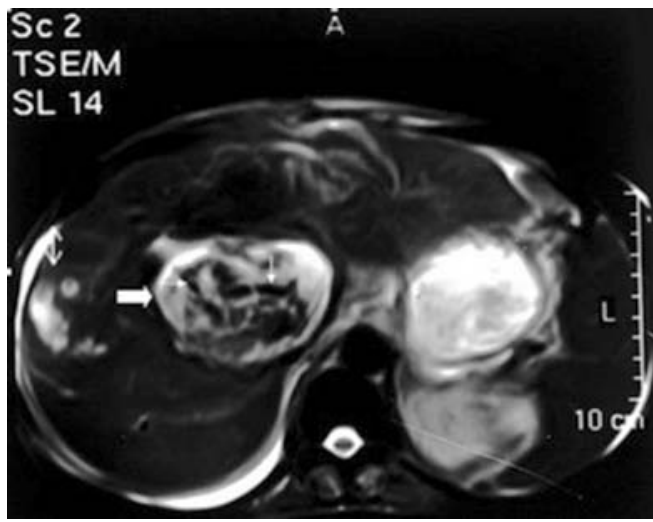

Figure 1B. A 37-year-old woman with HDL and BCS presented with right upper abdominal pain and fever. TransverseT2-weighted MR image shows hydatid cyst (thick arrow) with multiple hypointense linear and band like structures (thin arrows) representing detached and degenerated membranes. Also noted is focal disruption of the hypointense band at the anterior aspect of the hydatid cyst, suggesting rupture.

spective study is to report a group of patients who developed BCS secondary to HDL, with the special emphasis on the sonographic appearances of typical intrahepatic venous collaterals that aid in the correct diagnosis.

\section{Patients and methods}

Thirteen patients ( 8 women, 5 men, age range 16-71 years, mean age 38 years) who were diagnosed with HDL and BCS at US within an eight-year period were included in this study. Videotape records or videoclips stored in the hard drive of the US scanner including both gray-scale and color Doppler examinations were retrospectively evaluated by two radiologists with at least 10 years experience in abdominal CDUS. Both radiologists evaluated the examinations independently. In case of discordance, interpretations were performed by the consensus review.

Color Doppler examinations were evaluated for the presence of the following intrahepatic venous collaterals which are 


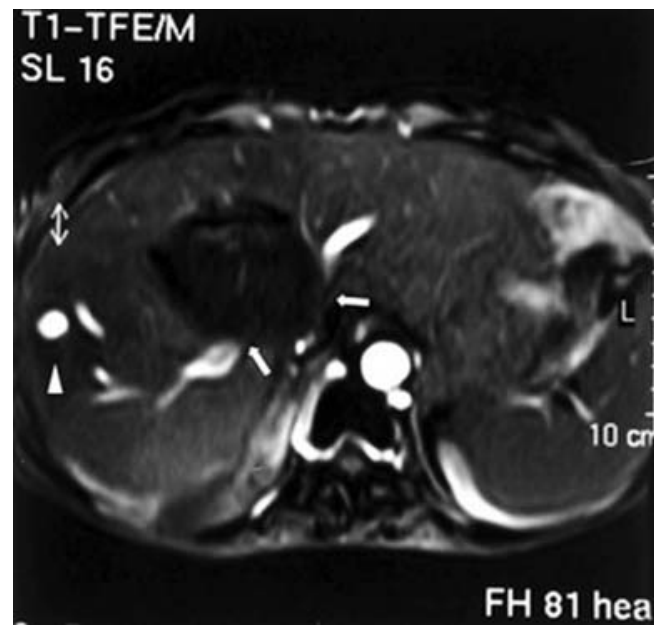

Figure 1C. A 37-year-old woman with HDL and BCS presented with right upper abdominal pain and fever. Transverse T1-weighted contrast-enhanced MR image also reveals the large subcapsular collateral (arrowhead). Both the left and right hepatic veins (arrows) are compressed at the hepatic venous confluence. At surgery, an infected hydatid cyst with intrahepatic rupture was found.

considered almost typical for BCS: (1) subcapsular collateral veins, (2) comma-shaped veno-venous collateral veins, (3) fragmented veins, (4) spider-web veins and (5) linear veins arranged in a non-anatomical distribution. ${ }^{7,14,15}$ In addition, the localization and relationship of the hydatid cyst with regard to the hepatic veins and/or suprahepatic IVC were also evaluated. HDL was sonographically classified according to an international consensus classification proposed by WHO. ${ }^{16}$ According to this classification, the cystic lesion (CL) represents an unilocular cyst with no visible cyst wall, type1 cystic echinococcosis (CE)1 represents unilocular cysts with visible cyst wall, type CE2 represents multivesicular cysts with daughter cysts, type CE3 represents cysts with floating membranes or complex hydatid cysts with a semisolid appearance, type CE 4 represents hydatid cysts with a heterogeneous hypoechoic appearance and finally type CE 5 represents hydatid cysts

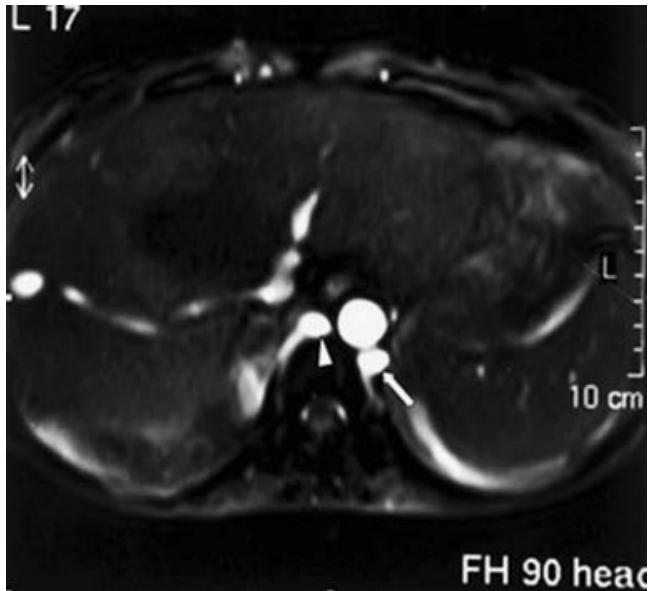

Figure 1D. A 37-year-old woman with HDL and BCS presented with right upper abdominal pain and fever. TransverseT1-weighted contrast-enhanced MR image at a slightly different level than Figure1-C demonstrates the dilated azygos (arrowhead) and hemiazygos veins (arrow) which act as extrahepatic collaterals to provide drainage for obstructed hepatic venous flow. These collaterals could not be demonstrated at US.

with thick calcified walls. Because of their nonspecific US appearances, CL's were not included in this present study. The mean diameter of the hydatid cysts as measured at US was $12.2 \mathrm{~cm}$ (range, 6-21 cm), all located at the hepatic dome. Based on WHO classification, one of 13 of our patients $(7.7 \%)$ had type CE1, six (46.2\%) patients had type CE2, two (15.4\%) patients had type CE3, three patients (23.1\%) had type CE4 and the remaining patient had type CE5.

Gray-scale and CDUS examinations were performed either with a Toshiba SSA 270 A (Tokyo, Japan) unit or GE Healthcare (Milwaukee, WI) LOGIQ 9 machine equipped with $3.75 \mathrm{MHz}$ and $5-12 \mathrm{MHz}$ broadband transducers, respectively.

All MRI studies were performed on a 1.5T MRI unit (Gyroscan Intera, Philips Medical Systems, Netherlands). Nonenhanced T1 and T2 -weighted images and dynamic contrast-enhanced MR images of the liver were obtained in 10 patients. 


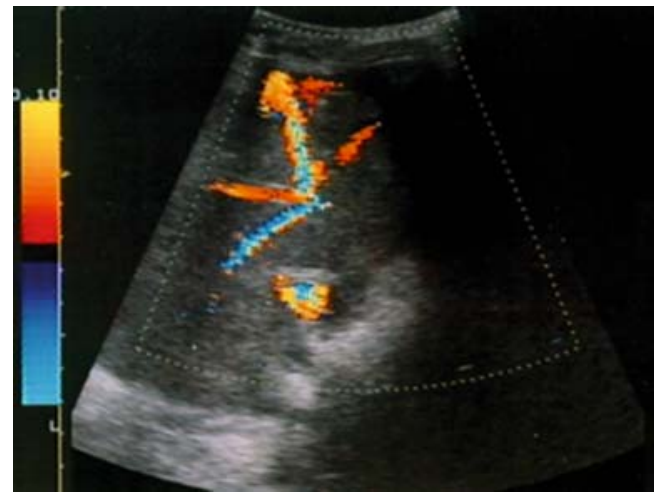

Figure 2A. A 43-year-old man with BCS and HDL presented with nonspecific right upper abdominal pain. Transverse color Doppler sonogram obtained in right intercostal plane and showed collateral veins suggesting BCS. Poor patient cooperation and extensive gasseous distention hampered both the evaluation of the major hepatic veins and the hydatid cyst.

MR images were evaluated for (1) the localization and relationship between the hydatid cyst and hepatic veins and/or suprahepatic IVC, (2) patency of the major hepatic veins and/or suprahepatic IVC and (3) presence of intrahepatic and extrahepatic collateral veins.

The reason for the referral was to rule out BCS in 10 patients. Clinical findings included hepatomegaly (9 patients) splenomegaly (3 patients), lower extremity swelling (2 patients), ascites (8 patients), and superficial abdominal collaterals (4 patients). Oesophageal varices were evident endoscopically in 4 patients. In the remaining 3 patients, the clinical presentation was nonspecific including abdominal fullness, right upper abdominal pain and fatique.

\section{Results}

During the retrospective analysis of sonographic data, at least one type of intrahepatic venous collateral typical for BCS was detected in all of our 13 patients with HDL and BCS. At CDUS, five different types

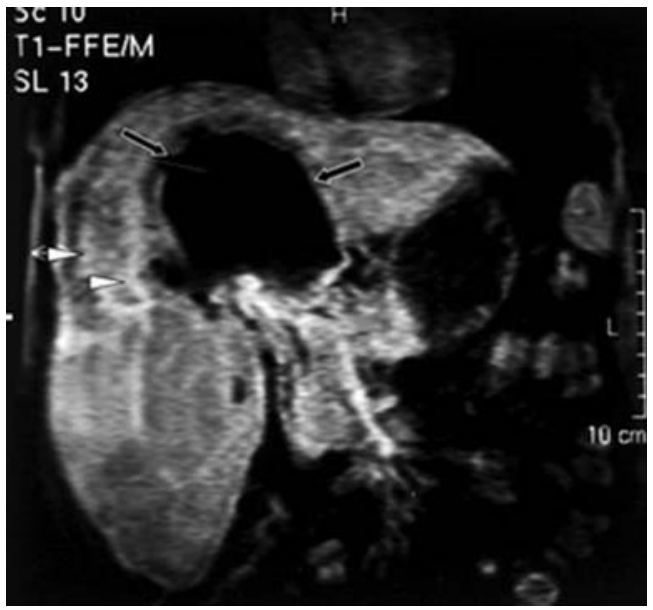

Figure 2B. A 43-year-old man with BCS and HDL presented with nonspecific right upper abdominal pain Coronal T-1 weighted contrast-enhanced MR image reveals both the hydatid cyst (arrows) and collateral veins (arrowheads).

of intrahepatic venous collaterals including subcapsular veins (Figure 1a), comma -shaped veins which sometimes resemble a hockey-stick (Figures 2c, 2d), spider-web collaterals (Figure 3a), fragmented veins (Figure $3 b$ ) and linear veins arranged in a non-anatomical distribution (Figures 2a, $4 b)$ were identified. The most frequently encountered collaterals at CDUS were comma-shaped veins which were seen in 12 of 13 (92.3\%) patients. Subcapsular collaterals were detected in 8 patients, linear veins arranged in a non-anatomical distribution were seen in 5 patients, spider web collaterals were encountered in 4 patients and fragmented veins were seen in 4 patients. In addition, at least 2 different types of intrahepatic collaterals were found in 8 of $13(61.5 \%)$ of our patients. In two patients, venous collaterals were not seen on gray-scale sonography but they were readily apparent at CDUS (Figure 4). All of the sonographically detected collateral veins demonstrated a monophasic-flat flow at Duplex Doppler US. At gray-scale US, a nonspecific heterogenous hepatic echo- 


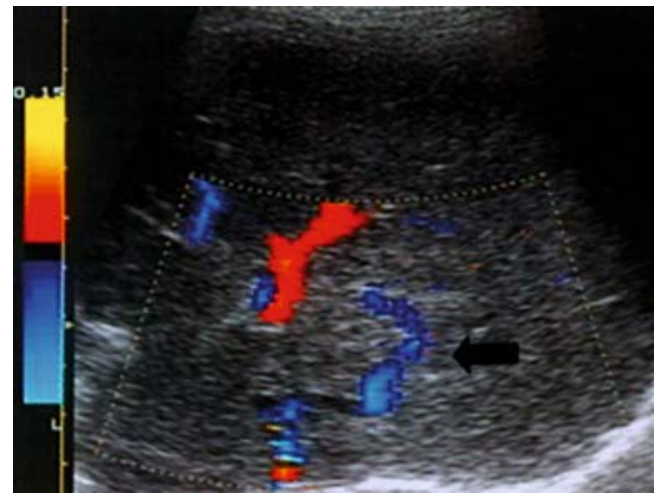

Figure 2C. A 43-year-old man with BCS and HDL presented with nonspecific right upper abdominal pain. Oblique subcostal color Doppler image depicts hockey-stick collateral (arrow) which drained direcly into the IVC.

pattern was observed in 8 patients. In 2 patients US showed a sharp demarcation between the anterior and posterior parts of the liver. The anterior part of the liver demonstrated diffuse hypoechogenicity with multipl coma-shaped venous collaterals, whereas the posterior part of the liver showed diffuse hyperechogenicity with no vascularisation at CDUS (Figure 5).

Only 3 different types of intrahepatic intrahepatic venous collaterals were visible at MR imaging. Subcapsular colllaterals were the most frequently detected collaterals at contrast - enhanced MR imaging and they were seen in 4 patients. Comma-shaped collaterals were detected in 3 patients, whereas, linear veins arranged in a nonanatomical distribution were observed in 2 patients. The spider -web collateral veins and fragmented collateral veins detected at CDS were not visible at MR imaging. No intrahepatic venous collateral could be identified in 4 of 10 patients who underwent MR imaging. On the other hand, MR imaging showed stenosis or severe compression of at least one major intrahepatic vein and / or suprahepatic IVC in all 10 patients for whom MR imaging was available (Figure 1c). At CDUS, however, stenosis or severe

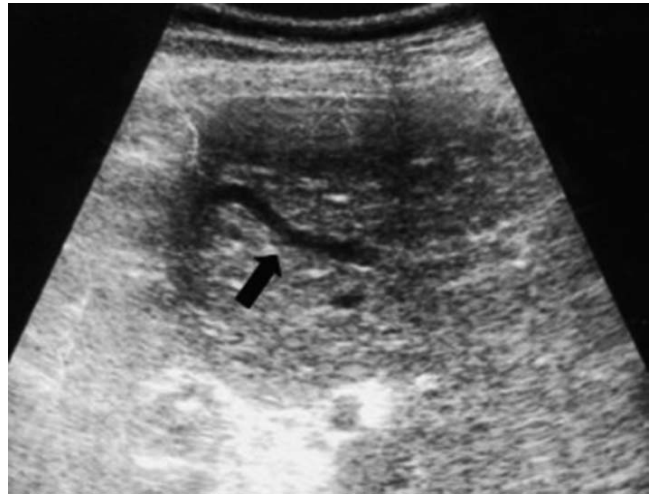

Figure 2D. A 43-year-old man with BCS and HDL presented with nonspecific right upper abdominal pain Transverse gray-scale sonogram obtained in the same patient reveals typical comma-shaped collateral (arrow) in a subcapsular location. At Duplex Doppler imaging, this vein demontrated monophasic flow (not shown). Comma-shaped collaterals are highly suggesitve, if not diagnostic of BCS.

compression of the major hepatic veins or suprahepatic veins could be demonstrated in 6 of $13(46.2 \%)$ patients.

Extrahepatic collaterals were seen in 5 of $10(50 \%)$ patients (Figure 1d) for whom MR imaging was available. No extrahepatic collaterals were identified at CDUS. In addition, neither CDUS nor MR imaging revealed portovenous collaterals. The portal vein was dilated (diameter more than 13 $\mathrm{mm}$ ) in 5 patients. Portal venous thrombosis was not observed in any patient.

\section{Discussion}

BCS secondary to HDL can occur if large hydatid cysts in the hepatic parenchyma compress the hepatic veins. To produce BCS, cysts have to be very large and lie in an appropriate position. Endoveinitis may also be a contributing factor to the development of BCS in HDL, especially in advanced stages.

As reported in a previous study, asymptomatic Budd-Chiari syndrome is not rare 


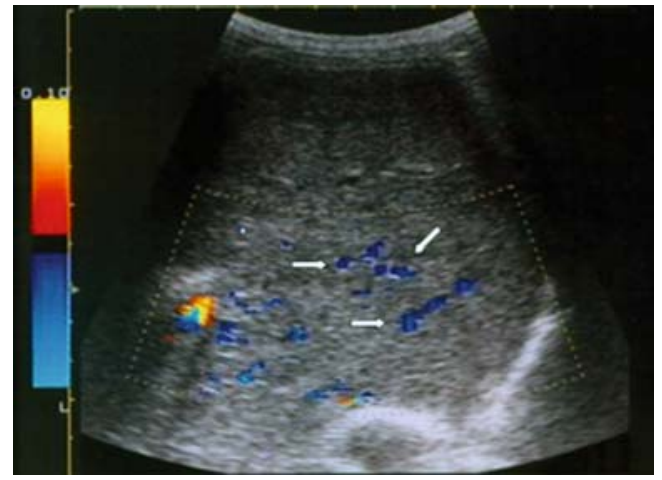

Figure 3A. A 44-year-old woman with BCS and HDL presented with upper abdominal pain. A unilocular hydatid cyst located at the hepatic dome was seen at gray-scale US (not shown). Oblique subcostal color Doppler image reveals spider-web collaterals (arrows).

and accounts for 15 to $20 \%$ of cases. ${ }^{17}$ Asymptomatic Budd-Chiari syndrome is associated with the spontaneous development of large intrahepatic and portosystemic collaterals.

Diagnosis of BCS based on direct US criteria may be difficult in patients even when the diagnosis is suspected clinically. Diagnostic difficulties at US include an inadequate evaluation of the major hepatic veins due to obesity, extensive bowel gas, cirrhosis, ascites and hepatomegaly. Intrahepatic venous collaterals are considered the most sensitive feature for the diagnosis of BCS, found in more than $80 \%$ of cases. Thus, familiarity with the sonographic appearances of these collaterals may aid in the correct diagnosis when the direct evaluation of the major hepatic veins is problematic at CDUS. Intrahepatic venous collaterals almost diagnostic of BCS were seen in all of our 13 patients at CDUS. ${ }^{7,14,15}$

In 2 of our patients who presented with the typical triad of BCS, US revealed an avascular, hyperechoic region in the peripheral, posterior aspect of the right hepatic lobe with a sharp demarcation zone (Figure 5). We believe that this appearance is analogous to the previously reported CT

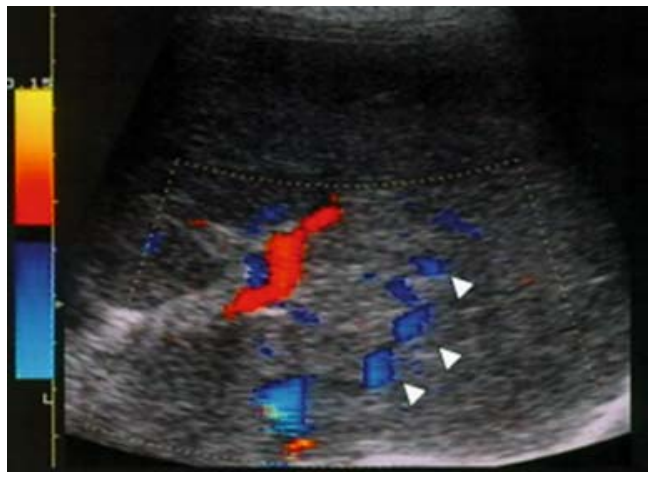

Figure 3B. A 44-year-old woman with BCS and HDL presented with upper abdominal pain. A unilocular hydatid cyst located at the hepatic dome was seen at gray-scale US (not shown). Color Doppler sonogram shows fragmented collateral veins (arrowheads). Both spider-web and fragmented collateral veins are almost diagnostic of BCS and may not be demonstrated at MR imaging, as it was in our case.

appearance of BCS and is related to congestion, edema and ischemia. ${ }^{14}$

Intrahepatic collateral veins resembling those seen in BCS have also been reported in Rendu-Osler-Weber syndrome, diaphragmatic hernia and congestive heart failure. However, in these clinical conditions collateral veins almost always show a pulsatile flow, whereas collateral veins in BCS typically demonstrate a monophasic flow at Duplex US. ${ }^{18}$

Extrahepatic collaterals were apparent in 5 patients at MR imaging (Figure 1d). None of these extrahepatic collaterals were visible at gray-scale US and MR imaging MRI seemed to be superior to US in this context.

HDL may not be a rare cause of BCS, especially where hydatid disease is endemic. Indeed, in a recent study which was done in an endemic area for hydatid disease, HDL was the second most frequent cause of BCS and constituted $8 \%$ percent of all patients with BCS. ${ }^{4}$

Our study has some limitations. Due to the limited number of patients with BCS and HDL, the statistical analysis was not performed. Nevertheless, US seemed to 


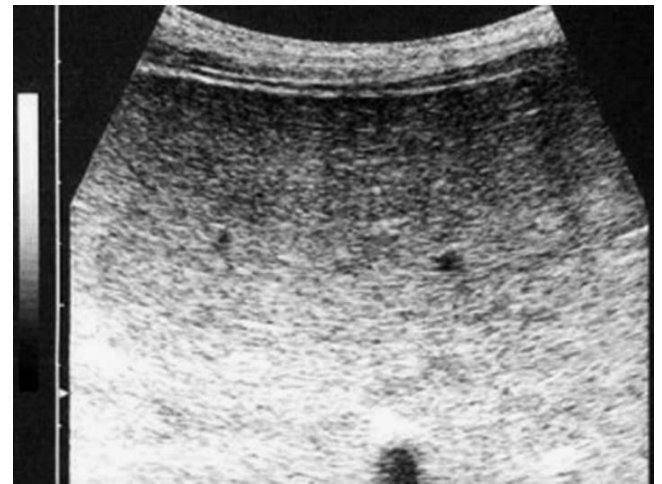

Figure 4A. Transverse magnified gray -scale sonogram of a 47-year-old man with BCS and HDL. The parenchymal echopattern appears heterogeneous. No apparent collateral vessel can be identified.

be superior to MRI in the demonstration of intrahepatic collaterals, whereas, MRI prevailed US in the demonstration of major hepatic venous or IVC involvement. Another limitation of our study is that the caudate lobe vein was not evaluated. We believe that the caudate lobe vein should be assessed during focused real-time examinations and retrospective evaluations may not be reliable to confirm or exclude dilatation of the caudate lobe vein.

In conclusion, our study shows that BCS secondary to HDL may not be exceptionally rare and may come as an unexpected finding during sonography. Familiarity with the typical sonographic appearances of the intrahepatic venous collaterals associated with BCS may enable the correct diagnosis and the prompt further treatment. We recommend CDUS to be performed in every patient with HDL regardless of the clinical presentation.

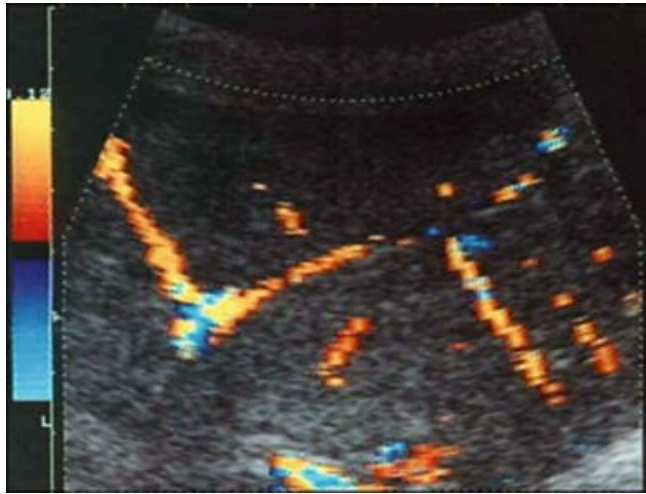

Figure 4B. Transverse magnified color Doppler sonogram of a 47-year-old man with BCS and HDL. At color Doppler imaging multipl intrahepatic collateral veins are easily seen.



Figure 5. A 35-year-old woman presented with severe right upper abdominal pain, hepatomegaly and ascites. Color Doppler image reveals a sharp demarcation zone between the anterior and posterior parts of the right liver lobe (arrows). Also noted is a totally calcified hydatid cyst (arrowhead). The anterior part of the liver is hypoechoic and contains multipl commashaped venous collaterals. The posterior portion of the right liver lobe demonstrates increased echogenicity, possibly secondary to hepatic congestion and/or edema. 


\section{References}

1. Erden A, Erden I, Karayalcin S, Yurdaydin C. Budd-Chiari syndrome: evaluation with multiphase contrast-enhanced three-dimensional MR angiography. Am J Roentgenol 2002; 179: 1287-92.

2. Kutlu R. Obliterative hepatocavopathy - ultrasound and cavography findings. Radiol Oncol 2008; 42: 181-6.

3. Janssen HL, García-Pagán JC, Elias E, Mentha G, Hadengue A, Valla DC; European Group for the Study of Vascular Disorders of the Liver. BuddChiari syndrome: a review by an expert panel. J Hepatol 2003; 38: 364-71.

4. Uskudar O, Akdogan M, Sasmaz N, Yilmaz S, Tola M, Sahin B. Etiology and portal vein thrombosis in Budd-Chiari syndrome. World J Gastroenterol 2008; 14: 2858-62.

5. Bedioui H, Nouira K, Ayadi S, Daghfous A, Bakhtri M, Ksantini R, et al. Budd-Chiari syndrome secondary to hepatic echinococcosis. Gastroenterol Clin Biol 2007; 31: 721-4.

6. Yildirim M, Engin O, Oztekin O, Akdamar F, Adibelli ZH. Diagnostic evaluation and surgical management of recurrent hydatid cysts in an endemic region. Radiol Oncol 2009; 43: 162-9.

7. Bargalló X, Gilabert S, Nicolau C, García-Pagán JC, Ayuso JR, Brú C. Sonography of Budd-Chiari syndrome. Am J Roentgenol 2006; 187: W33-41.

8. De BK, De KK, Sen S, Biswas PK, Das TK, Das S, et al. Etiology based prevalence of Budd-Chiari syndrome in eastern India. J Assoc Physicians India 2000; 48: 800-3.

9. Cox JS, Seymour AE, Clarkson AR. Hydatid disease of the liver associated with the Budd-Chiari syndrome. Aust N Z J Surg 1966; 35: 291-4.

10. Khuroo MS, Datta DV, Khoshy A, Mitra SK, Chhuttani PN. Alveolar hydatid disease of the liver with Budd-Chiari syndrome. Postgrad Med J 1980; 56: 197-201.

11. Emre A, Arioğul O, Alper A, Okten A, Uras A, Yalçin S. Hydatid cysts of liver and portal hypertension. HPB Surg 1990; 2: 129-33.

12. Khaldi F, Braham N, Ben Chehida F, Ben Jaballah $\mathrm{N}$, Bennaceur B. Hepatic hydatidosis and portal hypertension in children. Is it the Budd-Chiari syndrome? Ann Pediatr (Paris) 1993; 40: 631-4.
13. Vogel J, Görich J, Kramme E, Merkle E, Sokiranski $\mathrm{R}$, Kern P, et al. Alveolar echinococcosis of the liver: percutaneous stent therapy in Budd-Chiari syndrome. Gut 1996; 39: 762-4.

14. Chaubal N, Dighe M, Hanchate V, Thakkar H, Deshmukh H, Krantikumar R. Sonography in Budd-Chiari Syndrome. J Ultrasound Med 2006; 25: 373-9.

15. Brancatelli G, Vilgrain V, Federle MP, Hakime A, Lagalla R, Iannaccone R, et al. Budd-Chiari syndrome: Spectrum of imaging findings. Am J Roentgenol 2007; 188: W168-76.

16. WHO Informal Working Group. International classification of ultrasound images in cystic echinococcosis for application in clinical and field epidemiological settings. Acta Trop 2003; 85: 253-61.

17. Hadengue A, Poliquin M, Vilgrain V, Belghiti J, Degott C, Erlinger S, et al. The changing scene of hepatic vein thrombosis: recognition of asymptomatic cases. Gastroenterology 1994; 106: 1042-7.

18. Naganuma H, Ishida H, Konno K, Komatsuda T, Hamashima Y, Ishida J, et al. Intrahepatic venous collaterals. Abdom Imaging 1998; 23: 66-171. 